Tänzer, Sandra; Godau, Marc; Berger, Marcus; Mannhaupt, Gerd

\title{
Einführung in den Band
}

Tänzer, Sandra [Hrsg.]; Godau, Marc [Hrsg.]; Berger, Marcus [Hrsg.]; Mannhaupt, Gerd [Hrsg.]: Perspektiven auf Hochschullernwerkstätten. Wechselspiele zwischen Individuum, Gemeinschaft, Ding und Raum. Bad Heilbrunn : Verlag Julius Klinkhardt 2019, S. 9-15. - (Lernen und Studieren in Lernwerkstätten)

Quellenangabe/ Reference:

Tänzer, Sandra; Godau, Marc; Berger, Marcus; Mannhaupt, Gerd: Einführung in den Band - In: Tänzer, Sandra [Hrsg.]; Godau, Marc [Hrsg.]; Berger, Marcus [Hrsg.]; Mannhaupt, Gerd [Hrsg.]: Perspektiven auf Hochschullernwerkstätten. Wechselspiele zwischen Individuum, Gemeinschaft, Ding und Raum. Bad Heilbrunn : Verlag Julius Klinkhardt 2019, S. 9-15 - URN:

urn:nbn:de:0111-pedocs-201841 - DOI: 10.25656/01:20184

https://nbn-resolving.org/urn:nbn:de:0111-pedocs-201841

https://doi.org/10.25656/01:20184

in Kooperation mit / in cooperation with:

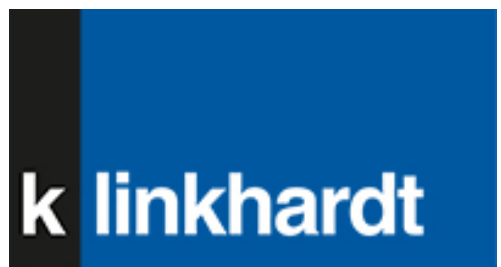

http://www.klinkhardt.de

\section{Nutzungsbedingungen}

Dieses Dokument steht unter folgender Creative Commons-Lizenz: http://creativecommons.org/licenses/by-nc-sa/4.0/deed.de - Sie dürfen das Werk bzw. den Inhalt unter folgenden Bedingungen vervielfältigen, verbreiten und öffentlich zugänglich machen sowie Abwandlungen und Bearbeitungen des Werkes bzw. Inhaltes anfertigen: Sie müssen den Namen des Autors/Rechteinhabers in der von ihm festgelegten Weise nennen. Dieses Werk bzw. der Inhalt darf nicht für kommerzielle Zwecke verwendet werden. Die neu entstandenen Werke bzw. Inhalte dürfen nur unter Verwendung von Lizenzbedingungen weitergegeben werden, die mit denen dieses Lizenzvertrages identisch oder vergleichbar sind.

Mit der Verwendung dieses Dokuments erkennen Sie die Nutzungsbedingungen an.

\section{Terms of use}

This document is published under following Creative Commons-License: http://creativecommons.org/licenses/by-nc-sa/4.0/deed.en - You may copy, distribute and transmit, adapt or exhibit the work in the public and alter, transform or change this work as long as you attribute the work in the manner specified by the author or licensor. You are not allowed to make commercial use of the work. If you alter, transform, or change this work in any way, you may distribute the resulting work only under this or a comparable license.

By using this particular document, you accept the above-stated conditions of use.

\section{Kontakt / Contact:}

\section{peDOCs}

DIPF | Leibniz-Institut für Bildungsforschung und Bildungsinformation Informationszentrum (IZ) Bildung

E-Mail: pedocs@dipf.de

Internet: www.pedocs.de

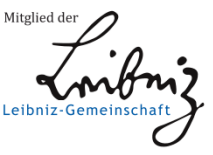




\section{Sandra Tänzer, Marc Godau, Marcus Berger und Gerd Mannhaupt}

\section{Einführung in den Band}

\section{Das Thema}

Unter dem Tagungsthema „Lernwerkstätten im Spannungsverhältnis zwischen Individuum, Gemeinschaft, Ding und Raum" wurde vom 18. bis 20. Februar 2018 an die Universität Erfurt zu einem breiten Diskurs über konzeptionelle Überlegungen, Forschungen und Praxiserfahrungen in und über Hochschullernwerkstätten eingeladen. Dieses Thema entspringt Überlegungen und Forschungsergebnissen des Forschungs- und Entwicklungsprojektes „Hochschullernwerkstatt“ unter Leitung von Sandra Tänzer und Gerd Mannhaupt. Dabei handelt es sich um eins von insgesamt fünf QUALITEACH-Projekten, die im Rahmen der Qualitätsoffensive Lehrerbildung vom Bundesministerium für Bildung und Forschung (BMBF) gefördert werden. Das Teilprojekt „Hochschullernwerkstatt“ nimmt speziell die Implementation und Wirkung hochschulischen Lernens in einer Lernwerkstatt in den Blick (Godau et al. 2018). An diesen drei Tagen im Februar 2018 waren knapp 100 Akteur*innen rund um die wissenschaftliche Auseinandersetzung um Hochschullernwerkstätten erschienen und in einen intensiven und gewinnbringenden Diskurs getreten.

Mit der Betonung von Individuum, Gemeinschaft, Ding und Raum wurden vier Kategorien respektive Themenfelder und Interdependenzbeziehungen aufgegriffen, die für die Lernwerkstatt Erfurt speziell, aber auch für Hochschullernwerkstätten allgemein konstitutiv sind.

Mit der Reflexion des Lernens von Individuen rücken die Adressat*innen des Lernens in den Mittelpunkt. Es geht um Fragen, welche Chancen und Herausforderungen Lernwerkstätten für die persönliche Entwicklung, Professionalisierung oder für die Initiierung subjektiver Erfahrungen bieten, wie auch um Fragen nach der Rolle von Lernwerkstätten im Kontext der eigenen Bildungsbiographie. Das fokussiert zunächst die Diskussion verschiedener Konzepte und Ansätze wie etwa erfahrungs-, problembasiertes, forschendes oder situiertes Lernen bis hin zu Fragen von Selbstbestimmung, Selbstständigkeit, Autonomie oder Agency. Darüber hinaus fallen hierunter auch je nach Diskurs ähnliche, aber in sich grundverschiedene Diskussionen um die anvisierten Bilder von Menschen, Zielgruppen oder Subjektadressierungen/-anrufungen in Lernwerkstätten. Das bietet nicht zuletzt 
Anlass für kritisch-reflexive Bezugnahmen auf Chancen und Grenzen, auf Inklusion und Exklusion oder Formen der Subjektivierung innerhalb der praktischen Umsetzung von einzelnen konzeptionellen Ansätzen und Prinzipien jener besonderen hochschulischen Lern- oder Bildungskontexte.

Gemeinschaft trägt sozialen Aspekten des Lernens in Lernwerkstätten Rechnung. So stellt sich doch immer wieder die Frage, wie Gruppenprozesse oder Lern- und Wissensgemeinschaften wie die sogenannten Communities of Practice (Lave \& Wenger 1991) nicht nur konzeptionell bedacht, sondern empirisch beobachtet und gefördert werden können. Gedacht sei etwa an kooperativ-arbeitsteilige oder kollaborativ-gemeinsame Formen kollektiven Lernens und solche sich anknüpfenden Überlegungen etwa zur Spezifik von Rollen Lehrender und Lernender innerhalb von Lernwerkstätten oder die Vernetzung innerhalb und außerhalb der jeweiligen Hochschule im Sinne einer Verzahnung formaler und informeller Lernkontexte.

$\mathrm{Zu}$ den Dingen: Seit jeher zeichnen sich Lernwerkstätten durch eine besondere, mitunter implizite Beachtung der Materialität (hochschul)pädagogischer Praxis vor allem mit Bezug auf phänomenologische und praxistheoretische Theorien aus (vgl. Stieve 2008). Lernwerkstätten an einzelnen Hochschulen erhalten ihre Individualität ganz wesentlich durch die unterschiedlichsten Dinge. Diese bieten Anlass zur Auseinandersetzung mit ihnen und führen nicht zuletzt zur Frage: "Wohin mit all den erstellten Materialien?» Referiert wird damit auf den Status von Artefakten, Medien, Technologien und physischen Objekten, die in Form von Einrichtungsgegenständen, Büchern, bereitgestellter oder durch Studierende erstellter Lehr-Lern-Materialien Ausgangspunkt von Erfahrungs- und Bildungsprozessen werden. Diskutiert wird das seit langem (wieder) etwa im Kontext einer „Pädagogik der Dinge“ (Nohl 2011) oder einer „Performativität der Dinge“ (Zirfas \& Klepacki 2013).

Und schließlich sind Lernwerkstätten Orte, physische Lokalitäten und soziale Räume des Lehrens und Lernens an Hochschulen. Berücksichtigt werden hiermit architektonische Qualitäten, die sowohl funktionale als auch ästhetische Besonderheiten in sich vereinen, und deren Bedeutung in der Situation als Lernumwelt, im Kontext von Raumdesign und Atmosphären, als Refugien (Wedekind \& Hagstedt 2011) oder „dritter Pädagoge“ (Müller-Naendrup 2013). Zur Diskussion stehen demgemäß das Verhältnis zwischen Raumgestaltung und Lernprozess respektive Fragen danach, wie sich im Raum unterschiedliche diskursive Praxen realisieren und Lernprozesse daran orientieren.

Im Nachgang an die Tagung haben wir das Tagungsthema „Lernwerkstätten im Spannungsverhältnis zwischen Individuum, Gemeinschaft, Ding und Raum “ für den vorliegenden Band ausgeweitet, um der Spannweite der hier versammelten Beiträge gerecht zu werden. Mit dem nun gewählten Titel des vorliegenden Bandes Hochschullernwerkstätten -Wechselspiele zwischen Individuum, Gemeinschaft, Ding 
und Raum soll hervorgehoben werden, dass nicht einzig Spannungsverhältnisse im Mittelpunkt der Betrachtung standen. Vielmehr werden gleichsam je nach Wechsel der Perspektive unterschiedliche Aspekte von Lehren, Lernen, Professionalisierung, Bildung usw. in Hochschullernwerkstätten beleuchtetet. Mit der Betonung des Wechselspiels wollen wir hier mehrere Wege öffnen: Es geht einerseits um innovative und kreative Auseinandersetzungen, die Spielzüge innerhalb konzeptioneller Verortungen neu oder anders denken, spielerische Lernformen in Lernwerkstätten oder Reflexionen der sozialen Regeln. Andererseits stehen die Artikel beispielhaft für Beschreibungen von Lernwerkstatt durch verschiedene Diskurse, mithin für damit verbundene sprachlich-kommunikative Eigenheiten. Das ermöglicht neue Perspektiven auf vielleicht gewohnte Bilder.

\section{Die Beiträge}

Der erste Teil des Bandes setzt sich mit konzeptionellen Grundlagen von und Impulsen für Hochschullernwerkstätten auseinander. Miriam Schöps, Dietlinde Rumpf und Kathrin Kramer werfen die grundsätzliche Frage nach dem (Selbst-) Verständnis von Hochschullernwerkstätten auf. Anknüpfend an die Ergebnisse der Befragung von Studierenden und Wissenschaftler*innen verschiedener lehramtsbezogener Fachdisziplinen der Martin-Luther-Universität Halle-Wittenberg sowie einer Expert*innendiskussion auf der Erfurter Tagung erläutern die Autorinnen sechs kennzeichnende Elemente, die mit Blick auf ihre Untersuchung den spezifischen Bedeutungsrahmen von ,Hochschullernwerkstatt' bilden. Ihr Ziel ist es, den Diskurs um ein gemeinsames Verständnis von Hochschullernwerkstätten voranzutreiben - eine Intention, an welche die beiden nachfolgenden Beiträge explizit anschließen. Axel Jansa, Lena S. Kaiser sowie Anna Jochums erweitern den Blick auf die Vielfalt von Hochschullernwerkstätten durch den Bezug zur Kindheitspädagogik. In ihrem Beitrag skizzieren sie Entstehungszusammenhänge, begriffliche Grundlagen sowie Merkmale ausgewählter Hochschullernwerkstätten in kindheitspädagogischen Studiengängen. Ihre Ausführungen münden in zehn zentrale Aspekte zur Kennzeichnung von Werkstattarbeit in der Kindheitspädagogik und der Einladung zu deren Diskussion. Mit der Frühpädagogischen Werkstatt an der Hochschule Emden/Leer steht im dritten Beitrag des ersten Teils dieses Buches eine konkrete Hochschullernwerkstatt der Kindheitspädagogik im Mittelpunkt. Dabei geht es im Beitrag von Edita Jung, Lena S. Kaiser und Ann-Christin Waldschmidt weniger um die Werkstatt selbst als um exemplarische Zusammenhänge, die jede Hochschullernwerkstatt betreffen, nämlich die Frage, wie Besuche von Kindern in hochschulischen Lernwerkstätten verantwortungsbewusst konturiert und begleitet werden können. Auf der Basis anerkennungstheoretischer Überlegungen umreißt der Beitrag ethische Grundsätze im Kontext der Einbe- 
ziehung von Kindern und reflektiert diese in ihrer Reichweite. Anregungen zur konzeptionellen Weiterentwicklung von Hochschullernwerkstätten unter Einbezug bislang im Werkstattdiskurs eher unbeachteter Theoriediskurse enthalten die nachfolgenden zwei Beiträge. Marek Grummt, Miriam Schöps und Marcel Veber diskutieren vergleichend Prinzipien von Lernwerkstattarbeit und kasuistischer Forschungswerkstatt und verweisen auf - für beide Ansätze fruchtbare - Synergiepotentiale, wenn beide Perspektiven innerhalb einer kasuistischen Lernwerkstattarbeit zusammengeführt werden. Der Beitrag von Ines Boban und Andreas Hinz lenkt den Blick auf den Diskurs um Demokratische Schulen und Demokratische Bildung. In ihren Ausführungen skizzieren sie das Selbstverständnis und die zentralen Grundlagen Demokratischer Bildung, um vor diesem Hintergrund Impulse für das Lernen in Hochschullernwerkstätten und die Professionalisierung von Lehrer*innen zu formulieren. In unmittelbarem Zusammenhang zum oben erwähnten Tagungsthema und dem Titel dieses Buches stehen die beiden letzten Beiträge des ersten Teils. Aspekte der Körperlichkeit bzw. Materialität, der Relationalität sowie der Ereignishaftigkeit und Vollzugshaftigkeit von Lernprozessen in ihrer räumlichen Situiertheit stehen im Mittelpunkt des Beitrages von Juliane Engel und Leopold Klepacki. Aus dieser praxeologischen und relationalitätstheoretischen Perspektive eröffnen die Autorin und der Autor einen Zugang zu Lernprozessen, die sie in den transaktionalen Gefügen zwischen Menschen, Räumen und Dingen verorten und leiten daraus Anforderungen an die Erforschung von Lernwerkstattarbeit ab. Der siebte und letzte Beitrag des ersten Teils bildet zugleich den Gelenkschritt in den zweiten Teil des Buches, indem sowohl konzeptionelle Überlegungen über den Raum und die Rolle von Menschen und Dingen als auch die Praxis von und in Hochschullernwerkstätten im Vordergrund stehen. Ausgehend von ihren unterschiedlichen Perspektiven als Forschende, Lehrende und Studierende in der OASE-Lernwerkstatt der Universität Siegen gehen Jochen Lange, Barbara Müller-Naendrup, Alina Schulte-Buskase und Jutta Wiesemann Fragen der näheren Bestimmung pädagogisch-konzeptioneller Merkmale von Hochschullernwerkstätten im Allgemeinen und der OASE-Lernwerkstatt im Besonderen nach und leiten daraus Anforderungen an deren Erforschung ab.

Der zweite Teil des Bandes gibt Einblick in die Praxis von und in Hochschullernwerkstätten. Franziska Herrmann reflektiert und untersucht am Beispiel der Seminarpraxis der "Didaktischen Forschungswerkstatt zum kreativen Schreiben“ an der TU Dresden nach der Methode der Grounded Theory, welche Irritation Studierende als selbstbestimmt Lernende erlebten, wie sie damit umgegangen sind und welche Rolle der Lernwerkstatt als Rahmen für forschendes Lernen beigemessen werden kann. Mareike Kelkel \& Markus Peschel wählen einen anderen empirischen Zugang, die Praxis von Hochschullernwerkstätten zu begleiten. Mit einer Fragebogenerhebung versuchen sie zu erfassen, inwieweit das auf Evaluation und Refle- 
xion angelegte Begleitseminar des Grundschullabors für Offenes Experimentieren (GOFEX_PP) an der Universität des Saarlandes als Lerngelegenheit, Chance oder Belastung wahrgenommen wird. Eva Franz kann in einem quasi-experimentellen Design erste positive Anzeichen für die Wirkung einer gezielten Intervention in der Lernwerkstatt auf die Kompetenz Studierender, geplante Lernaufgaben adaptiv zu gestalten, berichten. Nach der Planung und Durchführung von auf den Lern- und Entwicklungsstand fokussierten Interviews konnten die Studierenden der Interventionsgruppe die eigene Unterrichtsplanung besser mit den Lernausgangslagen der Kinder verknüpfen. Marcus Berger und Barbara Müller-Naendrup berichten von einem insofern besonderen Lernwerkstattzugang, als dass sie die didaktische Konzeption eines Kooperationsprojekts der OASE Lernwerkstatt der Universität Siegen und der Hochschullernwerkstatt der Universität Erfurt vorstellen. Das Lernwerkstattseminar setzt sich thematisch mit der Schnittstelle inklusiver Lernumgebungen und digitaler Medien mit besonderem Fokus auf kollaborative Zusammenarbeit auseinander, um das Lernen in Hochschullernwerkstätten mit dem Ansatz des Problem-Based Learnings (PBL) zu verknüpfen. Marc Godau und Sandra Tänzer gehen in ihrer qualitativen Studie der Frage nach, welche Bedeutung den Dingen im Hinblick auf Professionalisierung von Lehrer*innen beigemessen werden kann. Dafür verfolgen sie am Beispiel der Gestaltung einer „Drehscheibe“ im Kontext des Lernens in einer Lernwerkstatt an der Universität Erfurt die soziomaterielle Praxis des Entwicklungsprozesses eines Lernmaterials für Grundschüler*innen mithilfe der Grounded Theory. Abschließend beleuchten Ulrike Stadler-Altmann und Gerda Winkler eine gänzlich andere Art der Kooperation als Marcus Berger und Barbara-Müller-Naendrup. Sie beschreiben, wie an der Fakultät für Bildungswissenschaften in Brixen aus zwei Orten mit Objekten, einmal der Bibliothek mit ihrem Medienbestand und zum anderen der Lernwerkstatt mit ihren didaktischen Beständen, ein Raum wird, der zu Handlungen einladen soll. Erste empirische Einblicke weisen auf eine deutlich höhere Nutzung dieses neuen Raumes hin.

Der dritte Teil des Bandes bündelt unter der Überschrift Schnappschüsse aufHochschullernwerkstätten Posterbeiträge der Tagung. Alle Poster sind in größerer Auflösung und Farbe auch unter folgendem Link abrufbar:http://lernwerkstatt-erfurt. de/materialien-kiste/posterbeitraege/

Im Beitrag „doing AGENCY“ geben Pascal Kihm und Markus Peschel Einblicke in Öffnungsaspekte und -dimensionen beim Experimentieren im Sachunterricht anhand von „AGENCY“-Elementen. Dabei stehen insbesondere ein selbstbestimmter Lehr-Lern- bzw. Erkenntnisprozess sowie soziale und persönliche Offenheitsdimensionen im Fokus. Marcus Berger stellt in seinem Beitrag zum Thema „kollaboratives Problem-Based Learning“ die Konstruktion eines hochschuldidaktischen Settings vor, das sich zum Ziel setzt, mittels einer problembasierten und 
kollaborativen Struktur gezielt Aspekte professionellen Wissens zu fördern sowie die Transformation von verteilten in geteilte Wissensstrukturen zu unterstützen. Im Beitrag von Nikola Götzl und Magdalena Hollen „Die Lernwerkstatt Inklusion - Multiplikationsraum in Interdependenz mit Fortbildungsreihe" wird die Lernwerkstatt Inklusion der Universität Osnabrück als Multiplikationsraum für eine interdisziplinäre und inklusive Lehramtsausbildung im Rahmen der Fortbildungsreihe „Fit für den inklusiven Schulalltag“ vorgestellt. Im Fokus stehen hier klassische sonderpädagogische Förderbereiche, das Erstellen von individuellen Förderplänen sowie Inklusion und Kommunikation. In Sabrina Schudes Beitrag „Interdisziplinäre Kooperation zur innovativen Gestaltung von Unterricht und Lehre" wird die Kooperation von 17 Studienwerkstätten an der Universität Kassel im Rahmen einer interdisziplinären Ringveranstaltung zum Thema „Fremdheit“ dargestellt. Der Evaluationsansatz und erste Ergebnisse werden vorgestellt und ein Ausblick auf weitere Entwicklungen gegeben.

\section{Unser Dank}

Allen an diesem Buch mitwirkenden Autor*innen möchten wir herzlich für ihre Beiträge und für die konstruktive, sehr angenehme Zusammenarbeit bei der Manuskripterstellung danken. Wir danken ebenfalls Maria Trüpschuh für ihre sorgfältige Unterstützung bei der Vorbereitung der Druckvorlage und Thomas Tilsner und dem Team des Klinkhardt Verlags für die engagierte Beratung. Alle hier versammelten Beiträge haben wir mit Spannung, großem Interesse und anhaltendem Erkenntnisgewinn gelesen und laden Leser*innen ein, diese Erfahrung mit uns zu teilen. 


\section{Literatur}

Godau, Marc; Tänzer, Sandra; Berger, Marcus; Lingemann, Jana \& Mannhaupt, Gerd (2018): Studieren in der Lernwerkstatt. Erwartungen, Möglichkeiten und Herausforderungen für die Lehrer*innenbildung (nicht nur) in der Didaktik des Sachunterrichts. In: Franz, Ute; Giest, Hartmut; Hartinger, Andreas; Heinrich-Dönges, Anja \& Reinhoffer, Bernd (Hrsg): GDSU-Journal, H.8, 47-65.

Lave, Jean \& Wenger, Etienne (1991). Situated Learning: Legitimate Peripheral Participation. Cambridge.

Müller-Naendrup, Barbara (2013): Lernwerkstätten als „Dritte Pädagogen“. Räumliche Botschaften von Lernwerkstätten an Hochschulen. In: Coelen, Hendrik \& Müller-Naendrup, Barbara (Hrsg.): Studieren in Lernwerkstätten. Potentiale und Herausforderungen für die Lehrerbildung. Wiesbaden: Springer, 193-206.

Nohl, Arnd-Michael (2011): Pädagogik der Dinge. Bad Heilbrunn: Klinkhardt.

Stieve, Claus (2008): Von den Dingen lernen. Die Gegenstände unserer Kindheit. München: Wilhelm Fink.

Wedekind, Hartmut \& Hagstedt, Herbert (2011): Refugien? Über die Bedeutung von Lernwerkstätten in Schule und Hochschule. In: Grundschule, H. 6, 12-13.

Zirfas, Jörg \& Klepacki, Leopold (2013): Die Performativität der Dinge. Pädagogische Reflexionen über Bildung und Design. In: Zeitschrift für Erziehungswissenschaft, 16 JG., Sonderheft 25, 43-57. 